\title{
Corela
}

Cognition, représentation, langage

HS-12 | 2012

Langue, espace, cognition

\section{De la localisation à l'évaluation : des verbes préfixés évaluatifs au sens bien particulier}

\section{Dany Amiot}

\section{(2) OpenEdition}

\section{Journals}

\section{Édition électronique}

URL : http://journals.openedition.org/corela/2775

DOI : $10.4000 /$ corela. 2775

ISSN : 1638-573X

\section{Éditeur}

Cercle linguistique du Centre et de I'Ouest - CerLICO

\section{Référence électronique}

Dany Amiot, « De la localisation à l'évaluation : des verbes préfixés évaluatifs au sens bien particulier 》, Corela [En ligne], HS-12 | 2012, mis en ligne le 19 décembre 2012, consulté le 19 avril 2019. URL http://journals.openedition.org/corela/2775; DOI : 10.4000/corela.2775

Ce document a été généré automatiquement le 19 avril 2019

\section{(c) (i) (2)(2)}

Corela - cognition, représentation, langage est mis à disposition selon les termes de la licence Creative Commons Attribution - Pas d'Utilisation Commerciale - Partage dans les Mêmes Conditions 4.0 International. 


\title{
De la localisation à l'évaluation: des verbes préfixés évaluatifs au sens bien particulier
}

\author{
Dany Amiot
}

\section{Introduction}

1 Les prépositions sur et sous sont souvent analysées comme des prépositions fondamentalement spatiales (cf. par exemple Vandeloise 1986), même si elles peuvent bien sûr servir à exprimer d'autres types de relations; ces deux prépositions peuvent aussi avoir des emplois liés (surestimer, sous-alimenter). Dans ces emplois, les sens spatiaux, ou d'ailleurs les autres relations exprimées par ces prépositions, ne se retrouvent généralement pas ; apparaissent à leur place des sens que l'on peut qualifier d'évaluatifs ( surestimer, c'est «Déterminer par estimation un ordre de grandeur, une valeur qui excèdent la grandeur réelle, la valeur réelle", et sous-alimenter «Utiliser de façon insuffisante par rapport aux ressources productives $»^{1}$ ).

2 Cet article a pour premier objectif de montrer comment a pu s'opérer le passage de la préposition au préfixe et, ce faisant, de la localisation à l'évaluation. Nous verrons cependant que l'évaluation construite par la préfixation est d'un type bien particulier; elle est de nature fondamentalement quantitative, cette particularité pouvant s'expliquer par la nature à l'origine prépositionnelle des préfixes.

Comme par ailleurs, il existe aussi une évaluation construite par la suffixation (travailloter, voleter), celle-ci sera étudiée dans un second temps, ce qui permettra de montrer qu'elle est d'un type tout à fait différent de l'évaluation préfixale : elle n'est pas quantitative mais qualitative, dans la mesure où elle modifie le concept attaché au lexème de base (cf. infra, § 3.2.). 
Comme les quelques exemples cités le montrent, cette étude sera menée sur les verbes construits sur base verbale ; c'est en effet cette catégorie, la moins étudiée, qui paraît la plus à même de pointer les différences entre les deux types d'évaluation.

\section{Morphologie évaluative}

Dans l'évaluation morphologique, le sens du dérivé se calcule tout à fait classiquement en tenant compte du sens de la base, mais le calcul se fait d'une manière particulière : il met toujours en jeu une norme, quelle qu'elle soit; ainsi dire de quelqu'un qu'il est sousalimenté, c'est dire qu'il n'est pas suffisamment alimenté, ceci par rapport à une norme, explicite ou non.

6 Par ailleurs, d'un point de vue typologique, Grandi \& Montermini (2005) ont noté un phénomène intéressant: l'évaluation morphologique peut être considérée comme une exception par rapport au modus operandi morphologique classique : en effet, lorsqu'une langue possède des préfixes et des suffixes, il existe généralement une sorte de partage des tâches : ce qui est pris en charge par la suffixation - par ex. en français la formation des noms déverbaux d'action (développer / développement, accrocher / accrochage, évaluer / évaluation, etc.) ou des noms désadjectivaux de propriété (beau / beauté, plein/ plénitude, colère / colérique, etc.) - ne l'est pas par la préfixation et inversement; ainsi par exemple en français seule la préfixation peut construire des lexèmes à sens négatif (possible/ impossible, rythme / arythmie, héros / antihéros, etc.) ou à sens localisateur (cadre / encadrer, etc.).

7 Or, le seul domaine qui contrevienne de façon massive à ce partage des tâches est le domaine de l'évaluation, et ceci dans de nombreuses langues, notamment les langues indo-européennes, mais pas uniquement (cf. Grandi \& Montermini 2005) ${ }^{2}$. En effet, si comme Grandi (2002) on considère que la morphologie évaluative construit des lexèmes sur la base de deux oppositions : l'opposition SMALL $\leftrightarrow$ BIG (PETIT $\leftrightarrow$ GRAND) mise en jeu par l'évaluation quantitative; et l'opposition GOOD $\leftrightarrow$ BAD (BON $\leftrightarrow$ MAUVAIS) mise en jeu par l'évaluation qualitative, alors il est facile de constater que la préfixation comme la suffixation peuvent exprimer ces valeurs; les exemples du français le confirment amplement $^{3}$ :

small $\leftrightarrow$ big

(1) Diminution préfixale : micro- (organisme / micro-organisme)

Diminution suffixale : -et (livre / livret)

Augmentation préfixale : méga- (concert / méga-concert)

Augmentation suffixale: n'existe pas en français, mais cf. en italien: -one (palla

'balle' / pallone 'ballon's)

$\operatorname{good} \leftrightarrow$ bad

(2) Mélioration préfixale : super- (héros / super héros)

Mélioration suffixale : -issime (excellent / excellentissime)

Péjoration préfixale : sous- (homme / sous-homme)

Péjoration suffixale : -asse (marron/marronnasse

Que la morphologie évaluative construise dans une même langue le même type de sens par les deux opérations que sont la préfixation et la suffixation est un fait relativement étonnant en soi, mais si j’y fais référence ici, c'est parce que cette dualité me servira à montrer ce que l'évaluation préfixale construite par des préfixes d'origine prépositionnelle à capacité localisatrice a de particulier et de différent par rapport à l'évaluation suffixale, dont les suffixes ne possèdent pas une telle origine. 


\section{La préfixation évaluative}

\subsection{De la préposition au préfixe}

Il existe de très nombreux liens entre la catégorie de la préposition et celle du préfixe, et dans une langue comme le français, les préfixes seraient à $87 \%$ d'origine prépositionnelle (selon les données du Robert Brio, cf. Amiot \& Montermini 2009). Sur et sous illustrent parfaitement un cas de grammaticalisation à partir de la préposition correspondante ${ }^{5}$, même si certains sont tentés de voir en sur et sous des prépositions, des adverbes ou des particules, i.e. des constituants syntaxiques, le mot composé étant alors généralement vu comme une sorte de proposition en raccourci (cf. par exemple Darmesteter 1894; Benveniste 1949).

Dans Amiot (2005), j'ai cependant essayé de montrer que sur est bien un préfixe (le même raisonnement pourrait être tenu pour sous) ${ }^{6}$, et qu'il est devenu tel au terme d'un processus de grammaticalisation (cf. aussi Amiot \& De Mulder 2002); voici quelques-uns des arguments :

- argument distributionnel: alors que les prépositions introduisent fondamentalement des catégories nominales ( $\mathrm{SN}$ : sur le fauteuil / sous la chaise, $\mathrm{N}$ : sur ordonnance / sous médicaments, Pronoms: sur lequel/sous laquelle, etc.), les préfixes ne s'adjoignent pas à des SN (une *surlacharge, un *sousl'officier), mais à des lexèmes, ceux-ci pouvant appartenir à des catégories lexicales différentes, des noms (surcharge/ sous-officier), des verbes ( surinterpréter/sous-estimer) et aussi, bien que plus rarement pour ces deux préfixes, des adjectifs (surpeuplé/sous-peuplé).

- argument sémantique : alors que la localisation spatiale est extrêmement fréquente avec les prépositions (sur la route, sous le fauteuil), elle est rare parmi les lexèmes construits (on en recense cependant quelques-uns : survêtement / sous-vêtement, surnappe / sous-nappe, etc.); ce sont les sens évaluatifs qui dominent, et de très loin, l'excès avec sur- (surcharge, surdoué, surévaluer), l'insuffisance avec sous- (sous-alimentation, sous-développé, sous-payer), sens qui ne sont que rarement exprimés par les prépositions correspondantes (ou les contextes dans lesquels celles-ci sont insérées).

- argument paradigmatique: si les arguments distributionnel et sémantique mentionnés distinguent les prépositions des préfixes correspondants, ils rapprochent ces derniers des autres préfixes évaluatifs, de hyper- et hypo- notamment (pour une justification, cf. Amiot 2005), avec lesquels ils entrent dans une sorte de relation paradigmatique; les lexèmes affixés par les différents préfixes illustrent le même type de sens :

(3) extra-fin / hyperfin / ultrafin / surfin 'extrêmement fin, plus fin que la moyenne'

11 Il nous semble acquis que sur/sous prépositions et sur/ sous formants de mots soient deux types d'entités de nature différente; qu'en est-il maintenant du lien entre localisation et évaluation?

\subsection{De la localisation à l'évaluation}

Même si le sens de localisation spatiale n'est bien évidemment pas le seul sens exprimé par les prépositions sur et sous, ce sens est fondamental et on peut se demander comment on est passé de ces sens prépositionnels localisateurs, à des sens préfixaux foncièrement évaluatifs. Une des hypothèses faites dans Amiot (2004a, 2005) est que la localisation 
comme l'évaluation mettent fondamentalement en jeu le même schéma interprétatif, à savoir: sur(-) comme sous(-) opèrent généralement dans des domaines structurés verticalement, ou qui peuvent être conçus comme tels (l'espace, la hiérarchie sociale, la quantité, etc. $\left.{ }^{7}\right)$, ce qui peut être représenté par un axe vertical orienté de bas en haut, du négatif vers le positif. La construction du sens du dérivé met en jeu une «cible " (ce qui est localisé) et un "site », le repère localisateur (dans les termes de Vandeloise 1986; «figure » et " ground » dans ceux de Talmy 1972) ${ }^{8}$; la localisation comme l'évaluation résultent d'une opération de repérage que l'on peut représenter pour sur de la façon suivante (pour sous, la structuration est identique mais l'opération de repérage se fait vers le bas / le négatif) :

Figure 1

\section{haut}

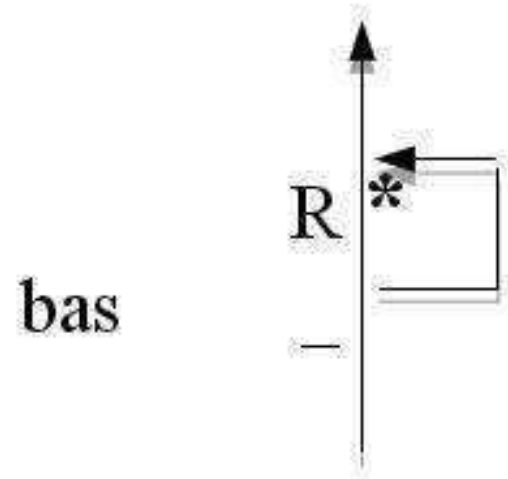

13 C'est le site (i.e. le régime de la préposition / le nom de base auquel s'adjoint le préfixe) qui joue le rôle de repère $(\mathrm{R})$.

Rien dans ce qui a été dit jusqu'à présent ne permet de distinguer la localisation de l'évaluation. Ce qui permet d'en rendre compte, c'est le fait qu'une préposition est un terme relationnel, ce que n'est pas un préfixe.

De façon générale, en effet, une préposition peut mettre en relation un site et une cible autonomes l'un par rapport à l'autre, ceux-ci étant d'ailleurs le plus souvent instanciés par des entités différentes, l'une et l'autre déterminées : il y a un chat sur le rebord de la fenêtre / une pièce sous la table / des fruits dans la coupe, etc.; c'est cette préexistence d'une cible et d'un site indépendants qui rend possible l'interprétation en termes de localisation 9. Dans l'évaluation préfixale, la cible et le site sont de même type (si tant est qu'il existe véritablement une cible), et ne sont actualisés par aucun déterminant: le dérivé (censé être la cible) dépend donc complètement, pour sa forme et pour son sens, de la base (le repère, i.e. le site) : une surcharge par exemple est fondamentalement une charge ${ }^{10}$. Comme charge peut dénoter une entité concrète, il serait bien sûr possible d'imaginer une interprétation en termes de localisation spatiale: une surcharge serait alors une charge située sur une première charge, mais ce n'est pas ainsi que surcharge est interprété, sans doute parce que cela ne répond à aucun véritable besoin de dénomination ${ }^{11}$. Dans la majorité des cas en effet, ce ne sont pas les paramètres «spatiaux » qui entrent en jeu pour effectuer le repérage nécessaire à la construction du sens du dérivé, mais une norme 
associée au terme de base (Rivara 1990, 1993); ce glissement de la localisation à l'évaluation permet de comprendre pourquoi sur-et sous- peuvent s'adjoindre non seulement à des noms concrets, mais aussi à des noms abstraits, des adjectifs ou des verbes.

Ce qui précède me conduit à faire deux courtes mises au point qui devraient donner un peu plus de poids au raisonnement, quelque peu lapidaire :

- La première concerne les adjectifs dénominaux qui, lorsqu'ils sont préfixés par sur ou sous, peuvent recevoir très facilement une interprétation spatiale; dans ce cas en effet, celle-ci met en jeu une cible (le $\mathrm{N}$ recteur de l'adjectif) et un site (le $\mathrm{N}$ de base de l'adjectif) différenciés et autonomes l'un par rapport à l'autre: une infection surrénale par exemple est une infection ( $\mathrm{N}$ recteur de l'adjectif, cible) qui se situe au-dessus du rein ( $\mathrm{N}$ de base de l'adjectif, site).

- La seconde mise au point est que, même en syntaxe, lorsque le site et la cible sont instanciés par des entités identiques non déterminées, l'interprétation en termes de localisation est moins facile; ce sont d'autres interprétations qui se font jour, selon le type de nom: l'itération, coup sur coup, souvent doublée d'accumulation, livre sur livre, ces séquences étant souvent intégrées à des constructions verbales, ainsi par exemple rendre coup sur coup, faire arrêt sur arrêt, lire livre sur livre, dire ânerie sur ânerie, etc.; ce type d'interprétation existe d'ailleurs avec d'autres prépositions : avancer pas à pas, rendre sou pour sou, fouiller pièce après pièce, etc. ${ }^{12}$

\subsection{Sur- et sous- préfixes évaluatifs : quelques généralités}

Comme les autres préfixes évaluatifs, sur- et sous- peuvent s'adjoindre à des bases de différentes catégories ( $\mathrm{N}, \mathrm{A}, \mathrm{V}$, il n'y a donc aucune contrainte d'unicité catégorielle) pour construire des lexèmes qui appartiennent généralement à la même catégorie que la base à laquelle ils s'adjoignent :

(4) sur- N/N charge / surcharge

A/A doué / surdoué

$\mathrm{V} / \mathrm{V}$ noter / surnoter

sous- $\mathrm{N} / \mathrm{N}$ continent / sous-continent

A/A calibré / sous-calibré

$\mathrm{V} / \mathrm{V}$ payer / sous-payer

17 Cette caractéristique de l'homocatégorialité (base et dérivé appartiennent à la même catégorie lexicale) n'est cependant pas surprenante vu que sémantiquement, le résultat d'une évaluation ne construit pas nécessairement un nouvel objet, différent de l'objet évalué, mais dit si celui-ci est conforme, ou non, à la norme.

Outre sur- et sous-, il existe bien sûr d'autres préfixes évaluatifs qui sont autant de formes concurrentes ; certains ont aussi une origine prépositionnelle mais ne correspondent plus à une préposition en français : super-, hyper-/ hypo-, extra-, ultra-, d'autres sont issus de la grammaticalisation d'autres catégories: adjectif (mini-/ maxi-, micro-, etc.), numéral ( méga-), etc. On notera que ces formes peuvent parfois se cumuler: une super méga teuf ${ }^{13}$. Cependant, sur- et sous- sont les seuls à pouvoir s'adjoindre de façon régulière et normée à des verbes pour former des verbes. ${ }^{14}$ 


\subsection{Le sémantisme des verbes préfixés}

19 Avec les verbes, nous retrouvons les mêmes types d'interprétations que ceux qui ont été mis en évidence au $\S 2.1$. lorsque nous avons opposé la préposition au préfixe: la préfixation en sur construit principalement des verbes qui s'interprètent avec un sens d'excès $(5 a)^{15}$ et celle en sous des verbes avec un sens d'insuffisance $(5 b)^{16}$ :

(5) a. surarmer, surestimer, surexploiter, surexposer, surgonfler, surpayer

b. sous-armer, sous-estimer, sous-exploiter, sous-exposer, sous-gonfler, sous-payer

Avec ces verbes, la norme qui sert de repère peut être parfaitement objective, par exemple avec des verbes comme sur-/sous-exposer, sur-/sous-gonfler, parce qu'on peut la chiffrer, comme ici au moyen d'une mesure temporelle, la minute, pour sur-/sous-exposer, ou d'une mesure de pression, le bar, pour sur-/sous-gonfler; dans d'autres cas elle pourra être plus subjective : selon moi, ce livre est sur-/sous-évalué ; je ne mettrais pas ce prix / à ce prix là il est vraiment donné, mais le repère en tant que tel sera cependant objectivisable (le prix à payer, au moins dans les cas simples). Comme le repère est généralement mesurable, l'évaluation tend à être quantitative ${ }^{17}$, ce qui d'ailleurs explique l'interprétation en terme d'excès / d'insuffisance.

Secondairement, on trouve aussi des verbes qui dénotent la répétition d'un premier procès :

(6) surcouper (en maroquinerie) «Couper une seconde fois pour obtenir la forme

définitive de la pièce »

surcontrer « Contrer (l'adversaire qui vient de contrer), au bridge. »

sursouffler « Opérer un nouveau soufflage (en métallurgie) »

On trouve principalement cette interprétation avec des verbes d'accomplissement ( surcouper), et des verbes d'achèvement (surcontrer), ce qui n'a en soi rien d'étonnant : ces verbes constituent en eux-mêmes des événements, dont les bornes permettent de concevoir le procès comme un second procès, réalisé à la suite d'un premier : ce «à la suite de » étant visiblement conçu, avec sur, comme un " en plus »; l'interprétation n'est donc pas conçue comme réellement temporelle et n'est pas incompatible avec ce qui a été dit du schéma interprétatif de sur lorsqu'il construit plus classiquement des verbes à sens évaluatif.

A cette interprétation peut être rattachée l'interprétation mi-itérative, mi-spatiale qui apparaît dans les verbes sous (7) :

(7) sursemer « Semer dans une terre déjà ensemencée »

surgreffer " Greffer à nouveau, sur une partie déjà greffée "

surbroder "Broder sur ce qui est déjà brodé »

La spécificité de ces verbes est qu'ils dénotent des procès dont l'action possède une incidence spatiale, l'itération peut donc se doubler d'une localisation, le second procès se déroulant au même endroit que le premier. Comme on le voit, itération et spatialité peuvent facilement être rattachés au schéma premier de la localisation et de l'évaluation (cf. fig.1).

Dans ces types d'interprétation, les procès sont d'une certaine façon pluralisés ; certains auteurs parlent dans ce cas de pluriactionnalité, dont Greenberg (2010) donne la définition suivante: "the phenomena where a certain derivational morphological marking on a verb (gemination, affixation, and many times partial or full reduplication), 
indicates that the event denoted by this verb is, in some sense, pluralized: repeated in time, distributed in various locations, holds of many participants, etc. ».

Depuis Cusic (1981), on distingue généralement deux types de pluralisation: la pluralisation externe (Event External Plurality) dans laquelle un même événement est répété, et la pluralisation interne (Event Internal Plurality), où la répétition concerne les sous-parties d'un seul et même procès ; refaire serait un exemple de la première, cligner un exemple de la seconde ${ }^{18}$. L'itération qui apparait dans les verbes préfixés de (6) et de (7) est bien évidemment de la pluralité externe : le verbe dérivé dénote deux occurrences du même procès ${ }^{19}$.

Dans le domaine verbal, d'autres interprétations peuvent être recensées en fonction du sémantisme de la base, mais ces cas sont rares. Les exemples sous (8) illustrent les différentes possibilités qui peuvent être rencontrées avec sur:

(8) a. surcomprimer " Techn. Augmenter la compression de (un gaz déjà comprimé) »

surélever « Accroître la hauteur de, donner plus de hauteur à »

b. surnager, survoler

c. survivre, surseoir « Vx Remettre pour un temps. », survenir «Vx Venir après, par

surcroît, s'ajouter à. / mod. Arriver, venir à l'improviste "

Il est en effet possible d'identifier une interprétation de supériorité quantitative mais non excessive en (8a), une interprétation purement spatiale en (8b), et une interprétation purement temporelle en $(8 \mathrm{c})^{20}$; ce dernier type d'interprétation n'est absolument plus productif à l'heure actuelle : les trois verbes mentionnés sont les seuls à s'interpréter de cette façon en français actuel et ils ont été, pour le premier, emprunté au latin ; quant aux deux autres, il étaient déjà attestés à la fin du XIème, au début du XIIème siècle.

Avec sous, il semble n'y avoir qu'une seule véritable interprétation non évaluative :

(9) sous-louer, sous-traiter, sous-diviser

dans laquelle on retrouve une forme d'itération, comme celle qui a été identifiée avec sur : sous-louer, c'est louer une seconde fois, non pas en tant que propriétaire, mais en tant que vrai ou « premier » locataire ; le même raisonnement peut-être tenu pour sous-traiter.

Quant à sous-diviser, qui est certainement un calque de subdiviser (peut-être refait par rétro-formation sous l'influence de sous-division), c'est là aussi diviser à nouveau ou opérer une nouvelle division; dans ce cas, ce qu'il y a d'un peu différent, c'est que cette interprétation met en jeu une relation de type partie/tout entre la base (qui opère sur le tout) et le dérivé (qui opère sur la partie) ${ }^{21}$.

Pour synthétiser rapidement ce qui vient d'être présenté à propos des sens des verbes préfixés par sur et sous, il a été montré que ces préfixes construisent très majoritairement des verbes qui s'interprètent avec un sens évaluatif quantitatif (excès / insuffisance). D'autres sens plus marginaux apparaissent aussi, le principal étant le sens itératif (pluralité externe); de plus, pour sur, nous avons relevé quelques verbes qui reçoivent une interprétation différente: supériorité quantitative, supériorité spatiale, interprétation temporelle. Cependant, ce qu'il y a d'intéressant, c'est que toutes ces interprétations peuvent se rattacher assez facilement à l'interprétation spatiale, sans doute l'interprétation la plus fondamentale de la préposition dont le préfixe est issu. Cela est particulièrement vrai de l'interprétation évaluative, celle qui nous intéresse réellement ici, mais cela est vrai aussi des autres interprétations. 


\subsection{La préfixation évaluative, la transitivité et l'aspect}

31 La préfixation évaluative paraît jouer un rôle minime en ce qui concerne la transitivité, et elle ne paraît jouer aucun rôle en ce qui concerne l'aspect. Je me contenterai ici de présenter les faits à propos de sur, mais ce qui est dit des mots construits par ce préfixe vaut pour ceux en sous.

i. Pour la transitivité, on remarque simplement que les verbes qui servent de base à la préfixation sont principalement transitifs (60 sur les 81verbes recensés dans le TLF et le $P R$ ), et quasiment tous sont issus de verbes eux-mêmes transitifs : la préfixation n'a, de ce point de vue (le seul qui nous intéressera ici) aucun impact particulier;

ii. Il en est de même pour ce qui concerne l'aspect lexical: les verbes dérivés sont généralement de même type aspectuel que les verbes de base (la répartition entre types de verbes est semble-t-il relativement classique) ${ }^{22}$; c'est ce que montre bien le tableau cidessous, qu'il sera intéressant de comparer au tableau 2 sous 3.2 .

Tableau 1 : Type aspectuel de la base et du dérivé (inspiré de Amiot \& Stosic $2011: 291$ )

\begin{tabular}{|c|c|c|c|c|c|}
\hline \multicolumn{2}{|c|}{ Verbe de base } & \multicolumn{4}{c|}{ Verbe dérivé } \\
\hline & & état & activité & accomplissement & achèvement \\
\hline état & 4 & 4 & & & \\
\hline activité & 30 & 1 & 28 & 1 & \\
\hline accomplissement & 32 & 1 & & 31 & \\
\hline achèvement & 15 & & & & 15 \\
\hline Total & 81 & 6 & 28 & 32 & 15 \\
\hline
\end{tabular}

32 La préfixation évaluative n'a donc pas pour effet de modifier le concept auquel renvoie le verbe de base, ce qui est cohérent avec ce qui a été affirmé plus haut, à savoir que le verbe de base sert simplement de repère sur une échelle et que l'évaluation consiste à localiser (au-dessus / en dessous), à quantifier (plus / moins, trop / pas assez), etc.

\section{L'évaluation suffixale}

\subsection{Généralités}

33 A première vue, l'évaluation suffixale possède beaucoup de points communs avec l'évaluation préfixale : il existe en effet ici aussi beaucoup de suffixes concurrents, et/te, ot, aille, ouille, on, ard, etc., ce qui donne parfois lieu à des phénomènes de cumul, par ex. dormichonner, dans lequel se trouvent associées les formes suffixales -ich- et -on(er)); la suffixation n'est pas non plus soumise au principe d'unicité catégorielle (les lexèmes de base peuvant être ds noms, des adjectifs ou des verbes) et les dérivés appartiennent généralement à la même catégorie lexicale que leur base (l'homocatégorialité se justifie de la même façon que pour la préfixation); ce que montrent bien les exemples sous (10) :

(10) - et/te N/N livre / livret

A/A simple / simplet

$\mathrm{V} / \mathrm{V}$ voler / voleter

-ot $\mathrm{N} / \mathrm{N}$ frère / frérot

A/A pâle / palot

V/V trembler / trembloter

-aille N/N fer / ferraille 
A/A dur / duraille

$\mathrm{V} / \mathrm{V}$ courir / courailler

-asse N/N paille / paillasse

A/A jaune / jaunasse

$\mathrm{V} / \mathrm{V}$ rêver / rêvasser Vendryès 1979, qui affirment : «Les suffixes indo-européens avaient rarement par euxmêmes un sens défini ; et il est le plus souvent impossible de décider d'où ils sont sortis " (§ 547)). A ma connaissance, il n'existe pas de travaux qui étudieraient systématiquement leur origine et le détail de leur évolution, cependant, on peut faire l'hypothèse que, même si ces suffixes possédaient une origine prépositionnelle comme sur- et sous-, ce qui est peu probable, celle-ci serait tellement éloignée dans le temps qu'elle ne serait sans doute plus opérante.

\subsection{L'évaluation dans les verbes suffixés}

37 Alors que la préfixation évaluative verbale ne concernait que sur et sous, la plupart des suffixes évaluatifs peuvent s'adjoindre à des verbes pour former des verbes. Outre les exemples qui apparaissent sous (10), il est encore possible de citer :

(12) -ailler : philosopher / philosophailler

-ouiller : cracher / crachouiller

-iller : croiser / croisiller

-onner : mâcher / mâchonner

-asser : rêver / rêvasser

La sémantique des verbes suffixés évaluatifs est très différente de celle des verbes préfixés ; bien que le sens du dérivé se construise aussi par rapport à une norme, il n'est pas possible de retrouver le sens très régulier d'excès / insuffisance construit par, respectivement, sur et sous. Pour mettre au jour l'irrégularité foncière qui caractérise l'évaluation suffixale, une série d'exemples contextualisés servira de point de départ:

(13) a. Il pleuviote depuis ce matin

Il lui a tapoté la main pour le réconforter

On lui a enlevé le plâtre et pour l'instant il marchote, disons qu'il a fait deux trois pas hier

Corela, HS-12 | 2012 
b. A peine sorti de la voiture, le chien s'est mis à courailler dans les bosquets

Ils ont discutaillé pendant un moment, puis se sont tus

Il ne fait que traînailler toute la journée sémantiquement, leur point commun ? S'il existe, il est difficile à appréhender : bien que portant le même suffixe, chaque verbe semble indiquer à sa manière que le procès dénoté par le dérivé n'est pas conforme à celui dénoté par la base: dans tapoter, ce qui semble caractéristique est que le procès 'taper' est réalisé plusieurs fois mais de façon moins intense, plus courte que lorsque l'on tape vraiment; il y aurait donc ici répétition et diminution d'une ou plusieurs propriété(s) de ce même verbe de base, à savoir l'intensité et la durée. De même, quand il pleuviote il ne pleut pas vraiment : la quantité de pluie est plus faible, la taille des gouttes, la force sans doute aussi. Vu le SP complément de temps, le procès occupe une certaine durée mais il n'est pas sûr qu'il occupe continument tout l'espace temporel : la pluie peut avoir été intermittente. Il semble donc y avoir aussi diminution d'une ou plusieurs propriétés du procès dénoté par le verbe de base (la taille des gouttes, l'intensité, la quantité de pluie) et aussi, éventuellement, une certaine répétition si le procès est intermittent, mais cela n'a rien d'obligatoire. Dans marchoter en revanche, au moins dans ce contexte précis, il n'est pas réellement question de répétition; le verbe suffixé nous dit simplement que le procès marcher n'est pas pleinement réalisé, seuls 2 ou 3 pas ont été faits, que l'on imagine bien peu assurés.

Ainsi, bien que les trois verbes soient issus de la même opération de suffixation, ils présentent d'assez grandes variations individuelles : la répétition ne se retrouve que dans deux d'entre eux, la diminution aussi, mais elle concerne à chaque fois des propriétés différentes; dans ces deux cas d'ailleurs, diminution et répétition paraissent liés, nous allons y revenir. Quant à l'interprétation de marchoter, il est possible qu'elle mette en œuvre la diminution (nombre de pas par rapport à ce que l'on appelle marcher?) mais cela n'a rien d'évident ; à nouveau, les propriétés (prototypiques ?) associées au procès marcher ne sont pas réalisées. Ces trois verbes issus de la suffixation évaluative illustrent bien un certain nombre de propriétés caractéristiques de ce type de verbes, à savoir : ce sont fondamentalement des verbes de manière, manière de déplacement (marchoter), manière de mouvement (tapoter), ou autre (pleuvioter) ; cf. sur ce point, cf. Stosic \& Amiot 2011. Il existe une grande indétermination dans la construction de leur sens et il est difficile, a priori, de prévoir quelles seront les propriétés affectées par la suffixation. Cela va être confirmé par la seconde série d'exemples.

41 Les verbes suffixés par aille sous (13b), font apparaître de nouvelles interprétations, qui ne sont pas plus unifiées par l'opération morphologique que les précédentes : lorsque le chien couraille, il court dans tous les sens, va à un endroit, à un autre en courant. En revanche, lorsque des gens discutaillent, de façon générale ils discutent un peu de tout et de rien, ou, comme le suggère le TLFi, «passe[nt] [leur] temps à discuter à propos de choses insignifiantes ». Quant à traînailler, il paraît signifier la même chose que traîner mais avec une nuance dépréciative plus marquée; nuance que possèdent généralement les verbes suffixés par aille.

Bien que les procès ici ne soint plus répétés ici, la pluralisation existe cependant, pluralisation de la trajectoire pour courailler, des sujets de discussion pour discutailler ${ }^{23}$. Si la diminution est encore présente, elle est liée à cette pluralisation : les lieux et les trajets sont nombreux mais courts, les sujets se succèdent mais sont de peu d'intérêt. Par contre, en ce qui concerne traînailler, aucune pluralisation, aucune diminution ne semble à

Corela, HS-12 | 2012 
l'œuvre, la suffixation paraît avoir pour seul but de marquer le terme pragmatiquement (dépréciation).

En résumé, l'absence de régularité typique de l'évaluation suffixale relève de plusieurs causes :

- aucun sens unique ne peut être associé aux lexèmes construits par un même suffixe (cf. les différentes interprétations associées aux lexèmes suffixés par ot ou par aille);

- un lexème dérivé cumule souvent plusieurs sens (cf. par ex. la diminution et la pluralité des procès dans tapoter) ;

- les mêmes sens se retrouvent avec les différents suffixes (la diminution par ex. apparaît dans les lexèmes construits avec ot et dans ceux formés avec aille).

Si cette absence de régularité distingue fondamentalement l'évaluation suffixale de l'évaluation préfixale, elle est cependant typique de l'évaluation suffixale en général ; cela a déjà été noté maintes fois, notamment à propos des noms suffixés, cette catégorie ayant été la plus étudiée, et de loin ; cf. uniquement pour le français Dal (1997, 1999), Delhay (1999), Fradin (2003), Fradin, Hathout \& Meunier (2003), Fradin \& Montermini (2009), etc. C'est d'ailleurs à cause de cette grande variété d'interprétations que certains, comme Delhay (1999), Fradin (2003), par exemple, ont pu distinguer deux grands pôles interprétatifs, le pôle référentiel (où l'on trouve la diminution notamment) et le pôle locuteur (dans lequel figurent par exemple les interprétations dépréciatives / mélioratives).

Cette variété d'interprétations est aussi mentionnée dans les travaux sur la pluriactionnalité verbale (cf. par ex. Cusic 1983 ; Lasersohn 1995; van Geenhoven 2005; Greenberg 2010 ; Tovena \& Kihm 2008, etc.) ; ainsi par exemple Greenberg (2010) note-t-il que « The variability is witnessed not only cross linguistically, but many times within the same language, and even with the same pluractional marker. » Cependant, ce phénomène a été peu étudié en français jusqu'à présent ; les travaux portant sur les verbes déverbaux sont en effet très peu nombreux (cf. tout de même Amiot \& Stosic 2011, Stosic \& Amiot 2011). L'un des effets de la suffixation évaluative verbale (cf. par ex. Grandi 2008 pour l'italien) comme d'ailleurs de la réduplication (cf. par ex. Greenberg 2010 pour l'hébreu) est effectivement la pluralisation du procès dénoté par la base.

Mais la pluralité construite par la suffixation évaluative est de la pluralité interne (et non de la pluralité externe comme avec les préfixes évaluatifs), que le verbe soit lui-même clairement pluriactionnel (cligner/ clignoter) ou non (mordre/ mordiller), sachant qu'il existe de nombreux verbes pour lesquels le fait d'être ou non pluriactionnel est relativement indécidable hors contexte (taper, sauter), les dérivés eux peuvent l'être sans difficulté : tapoter, sautiller.

Comme nous l'avons constaté, itérativité et diminution sont très souvent liées, la pluralisation du procès impliquant généralement une diminution des paramètres qui le constituent : ainsi par exemple voleter, pour prendre un autre suffixe, implique de petits mouvements rapides des ailes, des vols de courtes distances / durée. À nouveau les propriétés impliquées ne valent réellement que pour le procès dénoté par la base : la pluralité interne opère en effet sur la conceptualisation même du procès, c'est pour cela que l'évaluation suffixale a à voir avec la manière (Stosic \& Amiot 2011).

De ce fait, la suffixation évaluative est sensible à l'aspect des verbes :

i. elle exerce une contrainte préférentielle sur le type aspectuel des verbes de base, qui sont très majoritairement des verbes d'activité (cf. le Tableau 2), même si aucun type n'est réellement exclu ; 
ii. elle exerce aussi une contrainte sur l'aspect du verbe dérivé, qui dans ce cas aussi est majoritairement un verbe d'activité ; ce qui a pour conséquence que les verbes construits sur des verbes d'activité restent des verbes d'activité, que parmi ceux qui sont construits sur des accomplissements ou des achèvements, certains deviennent des verbes d'activité, cf. les exemples sous $(14 \mathrm{a}, \mathrm{b})$; seuls les états restent sysématiquement des états (14 c) :

Tableau 2 : Type aspectuel de la base et du dérivé (Amiot \& Stosic $2011: 291$ )

\begin{tabular}{|c|c|c|c|c|c|}
\hline \multicolumn{2}{|c|}{ Verbe de base } & \multicolumn{4}{c|}{ Verbe dérivé } \\
\hline & & état & activité & accomplissement & achèvement \\
\hline état & 11 & 10 & 1 & & \\
\hline activité & 138 & & 138 & & \\
\hline accomplissement & 8 & & 5 & 3 & \\
\hline achèvement & 14 & & 8 & & 6 \\
\hline Total & 171 & 10 & 152 & 3 & 6 \\
\hline
\end{tabular}

(14) a. achèvement $\rightarrow$ activité : piquer / picoter

b. accomplissement $\rightarrow$ activité : souper / soupailler, ranger / rangeailler

c. état $\rightarrow$ état : briller / brilloter ; vivre / vivoter

Les verbes dérivés sont donc fondamentalement atéliques, ce qui n'est pas étonnant : la correspondance entre pluralité et atélicité est en effet bien connue (cf. par exemple van Geenhoven 2004, 2005 ; cf. aussi Grandi 2008 pour l'aspect des verbes évaluatifs déverbaux suffixés de l'italien), mais cela vient étayer l'affirmation émise précédemment, à savoir que la suffixation opère bien sur le concept associé au verbe de base au sens où elle change son mode de réalisation.

\section{Une comparaison en guise de conclusion}

Si l'évaluation peut être construite par la préfixation comme par la suffixation, il semble bien, au terme de cette étude sur les verbes déverbaux, que les deux types d'évaluation soient vraiment très différents; pour le mettre en relief, les résultats vont être repris point par point :

i. d'un point de vue sémantique « global» :

les verbes construits pas préfixation sont très réguliers : en fonction du préfixe impliqué, ils expriment fondamentalement l'excès ou l'insuffisance; l'évaluation préfixale est quantitative ;

les verbes construits par suffixation sont irréguliers: les sens exprimés sont relativement indépendants des suffixes impliqués, et différents effets de sens se trouvent souvent intriqués: la diminution (celle-ci concernant des paramètres spécifiques au procès dénoté par le verbe de base), la pluralisation, le marquage pragmatique, etc. L'évaluation suffixale n'est en rien quantitative, elle est plutôt qualitative, d'où son affinité avec la manière.

i. bien qu'ils puissent l'un et l'autre construire des interprétations pluriactionnelles :

la pluriactionnalité construite par la préfixation, pluralité externe, est indépendante de l'évaluation ;

la pluralité construite par la suffixation, pluralité interne, est le plus souvent dépendante de l'évaluation (elle semble souvent être un effet de sens de la diminution pour la catégorie verbale).

i. en ce qui concerne l'aspect : 
la préfixation ne joue aucun rôle aspectuel : les verbes de base comme les verbes dérivés sont généralement de même type aspectuel ;

la suffixation a une incidence sur l'aspect: elle a effectivement une affinité très nette avec les verbes d'activité, soit parce que les verbes de base sont préférentiellement des verbes d'activité, soit parce qu'elle transforme en verbes d'activité un certain nombre de verbes qui ne l'étaient pas au départ. parce que relativement lexicalisée (cf. note 3 ); cela n'est pas le cas de l'évaluation préfixale, qui garde un lien vraiment étroit avec la localisation. Toutes les propriétés qui viennent d'être listées (évaluation quantitative, pluriactionalité externe, non prise en compte de la dimension aspectuelle de l'input comme de l'output) peuvent être attribuées au fait que ces préfixes s'originent dans des prépositions localisatrices; l'évaluation préfixale opère par le biais d'une norme qui reste extérieure au concept exprimé par la base - il joue simplement le rôle de repère (cf. figure 1) - alors que l'évaluation suffixale opère sur le concept lui-même, qui de ce fait est à la fois identique et différent par rapport au concept premier qui sert de norme, et c'est dans ces écarts par rapport à la norme ou au procès premier que se situe la manière.

Ces résultats doivent bien sûr être confirmés par des études à visée typologique pour voir si le lien entre localisation spatiale et évaluation se retrouve dans d'autres langues, que ce soit dans la formation des mots, ou dans d'autres domaines.

51 Je voudrais maintenant faire un rapide retour sur le statut ontologique de la figure 1, qui peut valoir pour la préposition comme pour le préfixe. Celle-ci peut être vue comme la représentation d'une sorte d'instruction procédurale sous-jacente à la construction du sens ou à l'interprétation de bon nombre de syntagmes ${ }^{24}$ et de mots dérivés, et ceci quel que soit le domaine (par exemple l'espace, le temps ou la hiérarchie pour la préposition; l'espace, l'excès pour le préfixe). Une telle vision ne suppose pas, en synchronie, une « primarité du spatial » (Groussier 1997), celle-ci étant certainement justifiée lorsque l'on adopte d'autres perspectives, diachroniques, acquisitionelles, ou autres.

\section{BIBLIOGRAPHIE}

Amiot, D., 2002. «Re- préfixe aspectuel ? », Cahiers Chronos 10, 1-20.

Amiot, D., 2004a. « Haut degré et préfixation », Travaux linguistiques du Cerlico 17, 91-104.

Amiot, D., 2004b. « Préfixes ou prépositions ? Le cas de sur-, sans-, contre- et les autres », Lexique $16,67-83$.

Amiot, D., 2005. «Sur- préposition et préfixe : un même sens instructionnel ? ", Revue de sémantique et de pragmatique 15/16, 101-119.

Amiot, D. \& De Mulder, W., 2002. « De l'adverbe au préfixe en passant par la préposition : un phénomène de grammaticalisation? », Lingvisticae Investigationes XXV/2, 247-273. 
Amiot, D. \& De Mulder, W., 2003. « Préposition contre préfixe », Recherches linguistiques 26, 203-232.

Amiot, D. \& Stosic, D., 2011. « Sautiller, voleter, dansoter : évaluation, pluriactionnalité, aspect », in E. Arjoca-Ieremia, C. Avezard-Roger, J. Goes, E. Moline \& A. Tihu (eds), Temps, aspect et classes de mots : études théoriques et didactiques. Arras : Artois Presses Université, 277-297.

Anscombre, J.-C., 1992. «Sur/sous : de la localisation spatiale à la localisation temporelle », Lexique $11,111-145$.

Benveniste, E., 1949. « Le Système sublogique des prépositions en latin », rééd. in Problèmes de linguistique générale 1. Paris : Tel-Gallimard, 1966, 132-139.

Corbin, D., 1999. « Pour une théorie sémantique de la catégorisation affixale », Faits de langues 14, 65-77.

Croft, W., 1990. Typology and Universals. Cambridge : Cambridge University Press.

Cusic, D.D., 1981. Verbal Plurality and Aspect. Thèse de doctorat. Stanford : Stanford University.

Dal, G., 1994. Un exemple de traitement associatif du lexique construit : analyse unificatrice des mots suffixés par et(te). Thèse de doctorat. Université de Lille 3.

Dal, G., 1997. Grammaire du suffixe -et. Paris : Didier Érudition.

Dal, G., 1999. « Suffixation par ette et bases verbales », in D. Corbin, G. Dal, B. Fradin, B. Habert, F. Kerleroux, M. Plénat \& M. Roché (eds), La morphologie des dérivés évaluatifs, Silexicales 2, Université de Lille 3, 37-47.

Darmesteter, A., 1894. Traité de la formation des noms composés dans la langue française. Paris : E. Bouillon éditeur.

Delhay, C., 1999. « "Diminutifs" et niveaux de catégorisation », Faits de langue 14, 79-87.

De Mulder, W. \& Flaux, N., 2005. «La préposition sur : essai d'analyse », in M. Tenchea (ed.), Prépositions et conjonctions de subordination : syntaxe et sémantique. Timisoara : Excelsior Art, 87-102.

Dendale, P. \& De Mulder, W., 1997. « Les traits et les emplois de la préposition spatiale sur », Faits de langues 9, 211-220.

Dendale, P. \& De Mulder, W., 1998. «Sur sur : réflexions sur l'emploi des ressemblances de famille en sémantique cognitive ", in G. Ruffino (ed), Atti del 21 Congresso internazionale di linguistica e filologia romanza, vol. 3 Lessicologia e semantica delle lingue romanze. Tübingen : Niemeyer, 213-222.

Dressler, W.U. \& Merlini Barbaresi, L., 1994. Morphopragmatics. Diminutives and Intensifiers in Italian, German, and other Languages. Berlin : Mouton de Gruyter.

Fradin, B., 2003. « Le traitement de la suffixation en -et », Langages 152, 51-77.

Fradin, B., Hathout, N. \& Meunier, F., 2003. « La suffixation en -et et la question de la productivité », Langue française 140, 56-78.

Fradin, B. \& Montermini, F., 2009. «La morphologie évaluative », in B. Fradin, F. Kerleroux \& M. Plénat (eds), Aperçus de la morphologie du français. Paris : Presses Universitaires de Vincennes, 231-266.

François, J., 2000. « Prépositions locales et grammaticalisation : les emplois non commutables ou sclérosés de sur », Travaux de linguistique du CERLICO 13/1, 231-255.

Goldberg, A., 1995. Constructions : A Construction Grammar Approach to Argument Structure. Chicago : University of Chicago Press. 
Grandi, N., 2002. Morfologie in contatto. Le costruzioni valutative nelle lingue del Mediterraneo. Milano : Francoangeli.

Grandi, N., 2008. I verbi deverbali suffissati in italiano. Cesena / Roma : Caissa Italia editore.

Grandi, N. \& Montermini, F., 2005. « Prefix-Suffix Neutrality in Evaluative Morphology », in G. Booij, E. Guevara, A. Ralli, S. Sgroi \& S. Scalise (eds), Morphology and Linguistic Typology, On-line Proceedings of the Fourth Mediterranean Morphology Meeting (MMM4). Catania, 21-23 Septembre 2003, Université de Bologne. URL : http://morbo.lingue.unibo.it/mmm/.

Greenberg, J.H., 1963. « Some universals of grammar with particular reference to the order of meaningful elements », in J.H. Greenberg (ed), Universals of grammar. Cambridge, Mass : MIT Press, 73-113.

Greenberg, Y., 2010. « Event Internal Pluractionality in Modern Hebrew: A Semantic Analysis of One Verbal Reduplication Pattern ", Brill's Annual of Afroasiatic Languages and Linguistics 2/1, 119-164.

Groussier, M.-L., 1997. « Prépositions et primarité du spatial : de l'expression de relations dans l'espace à l'expression de relations non-spatiales ", Faits de langues 9, 221-24.

Hickmann, M. \& Robert, S. (eds), 2006. Space in languages: Linguistic systems and cognitive categories. Amsterdam / Philadelphia : John Benjamins.

Kopecka, A., 2006. « The semantic structure of motion verbs in French : Typological perspectives », in M. Hickmann \& S. Robert (eds), 83-101.

Lakoff, G. \& Johnson, M., 1980. Metaphors We Live By. Chicago : Chicago University Press.

Langacker, R., 1987. Foundations of Cognitive Grammar, Vol I. Stanford, CA : Stanford University Press.

Lasersohn, P., 1995. Plurality, Conjunction and Events. Dordrecht : Kluwer.

Lehmann, C., 1985. « Grammaticalization: synchronic variation and diachronic change », Lingua e Stile 20, 303-318.

Meillet, A. \& Vendryès, J., 1924. Traité de grammaire comparée des langues classiques. 5 ème édition, 1979. Paris : Honoré Champion.

Norde, M., 2002. "Affixhood and beyond ", in I. Wisher \& G. Diewald (eds), New reflexions on grammaticalization. Amsterdam : John Benjamins, 45-65.

Rivara, R., 1990. Le système de la comparaison. Paris : Minuit.

Rivara, R., 1993. « Adjectifs et structures sémantiques scalaires », L'information grammaticale 58, 40-46.

Scalise, S., 1984. Generative Morphology. Dordrecht : Foris.

\section{NOTES}

1. Lorsqu'aucune indication n'est apportée, les définitions sont issues du TLF.

2. Il existe d'autres cas où la préfixation et la suffixation (et même d'ailleurs la conversion) entrent en concurrence ; cf. par exemple la construction de verbes sur base adjectivale (pauvre) appauvrir, banal / banaliser, grand / grandir), mais ils ne manifestent pas la régularité topologique de la morphologie évaluative. 
3. Et ceci, même si le français n'est pas une langue à morphologie évaluative développée, à la différence de l'italien par exemple. Ainsi, à un énoncé tel que :

ita. Oggi mangiamo (l'uov-etto + la carn-ina).

aujourd'hui mangeons (le oeuf-DIM + la viande-DIM) (ex. de Fradin $2003: 177$ )

correspondrait en français un énoncé où figurerait l'adjectif petit, qui jouerait le même rôle que les marques dérivationnelles de la diminution (DIM).

fra. Aujourd'hui, on va manger (son petit œuf / coco + sa petite viande).

4. Ballon a bien sûr été emprunté à l'italien, ce qui explique son sens particulier (i.e. augmentatif) par rapport aux autres noms construits par la suffixation en -on du français : glace / glaçon, lard / lardon, chat / chaton, qui tous ont un sens diminutif.

5. Il semble bien y avoir grammaticalisation dans la mesure où l'on retrouve dans cette évolution un grand nombre des paramètres caractéristiques de ce processus (cf. par exemple Lehmann 1985, Norde 2002): liage (bonding), décatégorialisation, changement sémantique, gradualité (gradualness), paradigmaticité, etc. ; en ce qui concerne les détails de certaines évolutions qui mènent de la préposition au préfixe cf. par ex. Amiot \& De Mulder 2002, Amiot (à par.).

6. Sur ce préfixe, cf. Corbin (1999).

7. On retrouve là les domaines de la structuration verticale pointés par Lakoff \& Johnson (1985: 25-27). En ce qui concerne l'espace, cela ne signifie pas bien entendu que toutes les relations spatiales nécessitent un repérage sur un référentiel structuré verticalement, mais que l'espace permet cette structuration, demandée par la préfixation en sur et sous.

8. "Localisé » et «localisateur » valent, il est vrai, fondamentalement pour l'espace, mais ces notions peuvent valoir / être transposées pour d'autres domaines, notamment celui du temps, de la quantité ou de l'évaluation, au moins l'évaluation quantitative, ce que nous allons voir par la suite.

9. Je me limite ici à la localisation spatiale; pour d'autres interprétations, cf. par exemple Amiot (2005).

10. Il est bien connu (cf. par exemple Scalise (1984), le premier à ma connaissance à avoir pointé cette particularité) que la préfixation homocatégorielle (lorsque le dérivé appartient à la même catégorie que la base) construit fondamentalement des lexèmes endocentriques, i.e. plus ou moins des hyponymes de la base.

11. Il ne semble pas exister en effet de réalité que l'on ait besoin de dénommer par un tel concept; en revanche, c'est ce même besoin de dénomination qui a permis de créer surveste ou sous-nappe, qui reçoivent bien ce type d'interprétation.

12. La structure V Ni prép Ni paraît de ce fait fonctionner comme une construction, au sens de Goldberg (1995).

13. On peut cependant se demander si nous sommes encore ici dans la préfixation ou si super et méga ne sont pas employés à la manière d'adjectifs / d'adverbes. Ce point ne sera pas abordé ici.

14. Dans les dictionnaires, aucune forme verbale en hyper-/ super- ou hypo- par exemple n'est attestée qui soit construite en français (les seules formes qui le sont ont été empruntées au latin ; cf. par ex. superposer, hypostasier, etc.). Sur la toile, il est possible de trouver des emplois du type j'ai hyper dormi / j'ai super aimé ce film (exemples fournis par mes rapporteurs) mais il n'est pas sûr que super ou hyper soient encore réellement des préfixes ici; ils semblent plutôt fonctionner comme des adverbes (auquel cas nous serions peut-être face à des cas de dégrammaticalisation) dans la mesure où hyper $+\mathrm{V}$ ne semblent pas former une unité lexicale : j'ai hyper bien dormi / super bien aimé ce film. De tels emplois nécessiteraient une autre étude.

15. Pour prendre un exemple restreint : sur les 81 verbes que l'on peut recenser dans le TLF et le Petit Robert, 36 reçoivent cette interprétation. Une telle tendance se retrouve dans les occurrences trouvées hors dictionnaires (par sondages effectués sur la Toile principalement). Ceci donne une première approximation, mais demandera à être confirmé par des études ultérieures plus approfondies. 
16. 13 sur les 21 verbes recensés (avec des principes de recension identiques à ceux de sur).

17. Au moins avec les verbes. Avec les adjectifs, elle peut être plus qualitative; cf. surdoué 'plus doué que la moyenne, extrêmement doué, mais le cas est rare avec sur/sous, cf. néanmoins suraiguiser « exacerber ». Avec certains verbes cependant (cf. surévaluer, surestimer), l'évaluation peut sembler à la fois quantitative et qualitative, mais cela est dû au sémantisme du verbe de base, dans lequel qualité et quantité sont déjà intimement liées.

18. Comme on le voit, il ne faut pas assimiler pluriactionnalité et morphologie (cf. à ce propos Amiot \& Stosic 2011).

19. Ces verbes illustrent bien des cas de pluralité externe: la répétition concerne deux occurrences de procès identiques, qui, ontologiquement, ne constituent pas un seul et même procès. La préfixation homocatégorielle, avec son corollaire l'endocentricité, permet en effet de construire des verbes dénotant des procès qui présupposent l'occurrence d'un premier procès ; cet effet de sens, que nous avons appelé ailleurs « effet de secondarité » (cf. Amiot \& De Mulder 2003), apparaît assez systématiquement lorsqu'il y a endocentricité, et ceci même avec des noms : une contre-manifestation par exemple est une manifestation (posé) faite pour s'opposer à une première manifestation (présupposé).

20. Les interprétations de $(8 \mathrm{a})$ et $(8 \mathrm{~b})$ sont compatibles avec le schéma interprétatif de la figure 1 ; l'interprétation temporelle des exemples sous (8c) l'est nettement moins : si l'opération de repérage permet bien de situer le procès exprimé par le verbe dérivé au-delà d'un repère, le plus souvent dénoté par le complément du verbe de base (par exemple survivre à quelqu'un c'est vivre au-delà de / après la mort de celui-ci), l'orientation de l'axe ne convient pas : l'axe temporel est généralement conçu comme un axe orienté horizontalement et non verticalement. Sans doute est-ce en raison de cette incompatibilité fondamentale que la préfixation à interprétation temporelle par sur / sous est très vite devenue improductive.

21. Cette interprétation méronymique se retrouve beaucoup plus fréquemment dans les noms que dans les verbes; il existe cependant quelques autres verbes, sous-catégoriser, sous-amender, auxquels correspond aussi un nom déverbal : sous-catégorie, sous-amendement.

22. Les verbes qui dénotent un état (par ex. abonder / surabonder) sont tous entrés dans la langue il y a très longtemps, et certains, comme survivre, ont été empruntés au latin. Par ailleurs, les deux verbes pour lesquelle la suffixation a opéré un changement aspectuel (activité $\rightarrow$ état: nager / surnager et accomplissement $\rightarrow$ état : faire / surfaire) ont été construits pour le premier, au XIVème siècle, et pour le second au XIème siècle.

23. Il est à noter que ces dimensions pluralisées sont reliées conceptuellement au procès, sans avoir à être exprimées sous forme de compléments, même si elles peuvent l'être : le chien se mit à courailler un peu partout / ils ont discutaillé une grande partie de la nuit de tout un tas de sujets.

24. Cela ne signifie bien sûr pas qu'elle vaille pour toute séquence introduite par sur; sur la polysémie de la préposition, cf. par exemple Dendal \& De Mulder (1997, 1998), Franckel \& Paillard (1998) ; François (2000), De Mulder \& Flaux (2005), etc.

\section{RÉSUMÉS}

En français, la morphologie évaluative peut être construite par deux opérations dérivationnelles différentes, la préfixation et la suffixation, les préfixes (super-, hyper-, sur-, hypo-, sous-, etc.) comme les suffixes (-et, -ot, -asse, -on, etc.) formant des ensembles relativement importants. Cet 
article cherche à montrer que l'évaluation construite par préfixation diffère fondamentalement de l'évaluation construite par suffixation, la spécificité de l'évaluation préfixale (évaluation quantitative : excès vs insuffisance) étant due au fait que les préfixes évaluatifs sont issus de prépositions locatives exprimant, au moins dans l'un de leurs sens, la supériorité / l'infériorité.

In French, evaluative morphology can result from two different derivational processes, prefixation and suffixation; prefixes such as suffixes forming important paradigms. This paper aims to show that the evaluation built up by prefixation is fundamentally different from evaluation built up by suffixation. The specificity of the prefixal evaluation (quantitative meaning: excess vs insufficiency) is due to the fact that evaluative prefixes originate into spatial prepositions whose at least one meaning express superiority / inferiority.

\section{INDEX}

Mots-clés : morphologie évaluative, préfixe, suffixe, évaluation quantitative, français

Keywords : evaluative morphology, prefix, suffix, quantitative evaluation, French

\section{AUTEUR}

DANY AMIOT

Université de Lille 3 - UMR STL 